\title{
HENRI BERR PAR LUI-MÊME
}

Dans les premiers mois de 1942, au plus sombre des jours de la sinistre occupation, au moment des persécutions raciales et des arrestations arbitraires, Henri Berr sentit la nécessité d'un retour sur lui-même, sur son passé, sur son œuvre. Il écrivit le texte qu'on lira ci-dessous, et qui devait, éventuellement, servir à sa défense.

A un âge avancé et en des temps d'épreuve, on dresse volontiers un bilan de sa vie.

Je suis dans ma quatre-vingtième année. Je suis né en Lorraine - à Lunéville - d'un père vosgien, d'une mere strasbourgeoise. Aussi loin que je puisse remonter dans le passé familial - plus de deux siècles - je ne trouve que Vosgiens et Alsaciens.

Mon trisaïeul paternel est né sous Louis XIV, ḋ Krautergersheim (Bas-Rhin); mon bisaieul, né en 1740, était inscrit le 13 septembre 1808 à la mairie de Raon-l'Etape avec ses huit enfants dont sept fils. Mon père, né en 1817, mort en 1870, était manufacturier à Lunéville : il avait pris - dés 1865 - l'initiative d'ouvres sociales en faveur de ses ouvriers et jouissait de l'estime générale. Je compte dans ma famille paternelle des magistrats, des ingénieurs, des artistes.

Du côté maternel, mon bisaieul a été inscrit a l'état civil de Reichshoffen le 19 octobre 1808, par le maire Héberlé, avec ses quatre enfants - dont l'ainé est mon grand-père. Celui-ci, né en 1801, mort en 1849, est une personnalité attachante. Il a joué à Strasbourg un róle important pour la propagation d'idées nobles, de sentiments humains. Il a créé un journal, La Pure Vérité, où, dans la tradition de notre pays, il s'associait ardemment à la « lutte incessante, comme il disait, entre la vérité et l'erreur», fier d'être né "en France, cette terre de gloire et de libertés. 
J'ai hérité de mon grand-père. Ce qui a animé toute ma vie spirituelle, avec la foi en la science, c'est l'amour de mon pays. La guerre de 1870, l'occupation de ma ville natale jusqu'en 1873, mon origine lorraine et alsacienne, tout a aiguisé en moi le patriotisme. A quinze ans, j'écrivais un drame, en vers, Pour la Patrie. Mon premier livre Vie et Science, se présentait sous la forme de lettres entre un « vieux philosophe strasbourgeois » et un étudiant parisien \. Après ma thèse de doctorat sur L'Avenir de la Philosophie, où je formulais mes croyances propres, fondées sur la libre recherche (1899), j'en ai résumé les idées dans un petit livre intitulé Peut-on refaire l'unité morale de la France? Pendant la guerre de 1914-1918, j'ai publié des - Paroles françaises \$. J'ai écrit des livres sur l'Allemagne : ils défendaient l'esprit français; ils étaient, cependant, assez objectifs pour que le juriste Friedich Grimm m'ait cité parmi les Français de la meilleure "compréhension».

Ma carrière universitaire. Je suis entré $6^{\circ}$ à l'Ecole Normale Supérieure en 1881, avec une dispense d'âge, après avoir obtenu au Concours général le prix d'honneur de Rhétorique (discours latin), le premier prix de discours français, le premier prix de Philosophie. J'en suis sorti agrégé des lettres, $4^{\circ}$, en 1884. Pendant quarante ans professeur de lycée, pendant vingt-neuf ans professeur de Rhétorique a Henri-IV, je crois avoir formé de bons Français. Mes élèves me sont restés fidèles. Beaucoup me font honneur.

Voici les témoignages que m'ont rendus le Directeur de l'Enseignement secondaire et, à la dernière distribution des prix où j'ai porté la robe, le Proviseur d'Henri-IV.

- Je savais, m'a écrit le directeur Vial le 3 mai 1925, que vous aviez pris la décision de nous quitter à la fin de l'année; et je le regrette. Mais je ne puis que m'incliner devant les raisons que vous me donnez. Vous continuerez à servir l'Université, dans une vie paralléle, et tout votre labeur sera encore un peu à nous. Je ne veux pas vous laisser partir sans vous remercier des bons services que vous avez rendus à tant de générations d'elèves et porter témoignage des éminentes qualités de votre enseignement. Permettez-moi d'ajouter aux remerciements du Directeur la reconnaissance du père de famille. Je suis heureux que mon fils, en cette année de Première, ait eu un tel professeur.s 
Et le proviseur Daux disait le 12 juillet :

«M. Berr était des nôtres depuis bientôt trente ans. Nommé professeur à Paris à vingt-cinq ans, il est entré au lycée Henri-IV comme professeur de Première dès l'année 1896. Il n'a jamais voulu quitter notre chère maison; il est resté fidèle d̀ sa classe, da ses élèves parmi lesquels il ne compte que des admirateurs et des amis. Il continue, d'ailleurs de s'intéresser à eux et de les suivre dans la vie longtemps après leur départ du lycée; il est resté pour beaucoup d'entre eux un guide, un conseiller, un ami véritable et sûr. Par sa distinction, par sa culture étendue et variée, il a fait honneur au lycée Henri-IV. Il ne sait pas se résigner au repos et il va maintenant consacrer toutes ses forces $\dot{a}$ "L'Evolution de l'Humanité », d cette belle ouvre qu'il a conçue, réalisée, qui prospère et grandit tous les jours entre ses mains habiles et contribue d étendre $\dot{a}$ l'étranger le prestige de la Science et de la pensée française. 》

En 1925 a commencé pour moi une vie nouvelle. Après avoir mené de front l'enseignement et la science, j'ai eu la chance qu'une santé et une curiosité d'esprit intactes m'aient permis alors d'inaugurer une carrière purement scientifique. J'adais créé la Revue de Synthèse historique en 1900, ¿L'Evolution de l'Humanité » en 1920 : j'ai créé en 1925 le «Centre international de Synthèse ».

Le président Doumer, passionnément, s'est intéressé a cette «Fondation pour la Science»: il en a été président, puis - appelé da l'Elysée - président d'honneur. Quand, en 1931, j'ai été promu officier de la Légion d'honneur, il a été mon parrain et il a tenu à en signer le diplôme de sa propre main. la même année, il était président d'honneur du Comité qui a fait frapper ma médaille da la Monnaie.

On sanctionnait ainsi, de façon émouvante, mes initiatives diverses.

Sur le rôle qu'a joué le Centre depuis 1925, sur l'influence qu'il a exercée, en France et au delà, sur les publications qui ont émané de lui, - les unes, hautement spéculatives, pour unifier les connaissances; les autres, Science et l' Encyclopédie périodique, pour les diffuser - j’ai recueilli de précieux témoignages.

J'en ai reçu même des Allemands, et même récemment. En 
aout 1940, il en est venu trois au Centre, pour l'inspecter : l'un d'eux a dit qu'il connaissait la maison. Ils n'ont rien dérangé, rien emporté - qu'une brochure qui reproduit ma médaille et les discours prononcés quand on me l'a offerte. Ils sont partis en souhaitant la continuation de nos travaux. Et nous avons continué, depuis deux ans. Depuis deux ans, \&L'Evolution de l'Humanité » suit son cours sous ma direction: en français, en espagnol, en anglais, en japonais, bientôt peutêtre en portugais, des millions d'exemplaires auront répandu dans le monde le goût et le modèle de la synthèse qui couronne le savoir. Et voici que cet Hymne à la Vie, roman d'idées, qui a hanté ma pensée pendant cinquante-cinq ans, vient de paraitre.

Dans cet exposé, je résume une longue vie : j'étale, non pas des titres d'orgueil, mais les services que j'ai tâché de rendre à mon pays. Ou, si j'ai quelque orgueil, c'est celui d'avoir été un bon Français, un pur Français. Sans y avoir de mérite, puisque toutes mes racines, de chair et d'esprit, sont dans la terre et dans la pensée françaises.

Il nous a semblé qu'aucun curriculum vitæ ne pouvait être meilleur que celui qu'Henri Berr avait rédigé lui-même.

Mais, après cette rédaction, de dures années devaient encore s'écouler, où les travaux du Centre de Synthèse devinrent de plus en plus clandestins, où les volumes de la Collection Descartes, «Pour la Vérité », furent mis au pilon, où les publications furent interrompues... Cependant, Henri Berr garda toujours sa sérénité et son optimisme fonciers, confiant dans la victoire de l'esprit sur les forces du mal. La Libération lui fit retrouver des ardeurs de jeunesse pour relancer revues, collections, Semaines de Synthèse, et reprendre ses propres travaux.

Victime d'un accident à quatre-vingt-trois ans, il surmontà cet handicap physique et, toujours avec la même lucidité, il poursuivit son œuvre, jusqu'au soir du 19 novembre 1954, où il s'éteignit doucement... comme une chandelle. 